\title{
Pathology confronts molecular targeted therapies
}

\author{
Jose Costa
}

Until very recently, pathological diagnosis combined with diagnostic imaging was used to guide therapy and predict prognosis for practically all patients with cancer. Histopathological classification and grading have been the major elements in determining patient management, and have largely been used to guide epidemiological, therapeutic and mechanistic studies by providing sufficiently robust criteria to classify neoplastic diseases. Now we can design specific methods to target dysfunction at the molecular level to induce cancer cell death, or to sensitize cancer cells to conventional therapeutic modalities. Molecular diagnosis of tumors enables the characterization of many of the pathogenetic abnormalities in 'cancer genes' that are present in tissue biopsies.

Molecular profiling of tumors, in conjunction with histopathology, is creating an increasingly robust molecular nosology of cancer; tumors can be now classified not only by their appearance (phenotypic classification) but also by the molecular lesion(s) that cause tumor cell development (mechanistic classification). As more structural and functional genetic alterations of cancer cells are discovered, and as technological advances improve the efficiency of clinical tests, the management and therapy of cancer patients is likely to become governed by molecular nosology. Patients with the same tumor histotype will receive a specific therapy only when the target molecular lesion is present and, perhaps, functionally relevant; patients with tumors arising at different sites and of different histotypes will receive the same drug if the same molecular lesion is present in both cancers. As the fields of basic research, drug discovery and diagnostics advance and converge, more and more patients will be treatable by molecular therapies, as already seen for chronic myeloid leukemia and gastrointestinal stromal tumors, among others. It is to be expected that cancers, kept in check by combinations of drugs targeting specific molecular defects, will frequently produce cells that 'escape' and cause tumor recurrence.
This scenario

requires

re-engineering

of pathology

laboratories...

by hitting the

right spot

and hitting it

hard we can

vastly improve

survival...

J Costa is an Advisory

Board member of

Nature Clinical

Practice Oncology.

Competing interests

The author declared he has

no competing interests.

www.nature.com/clinicalpractice doi:10.1038/ncponc0449
Tumors originate by a microevolutionary process and are constituted by heterogeneous populations of cells. These properties endow tumor cells with the capacity to adapt; far from being static, malignant tumors present biologically dynamic targets that can develop resistance to drugs. Pathological techniques capable of identifying the dynamic changes that occur in tumor cell populations need to be developed so that therapy can be optimized.

This scenario requires re-engineering of pathology laboratories. First, laboratories need to be staffed and equipped to process and handle cytology and tissue specimens in ways that ensure the applicability of sophisticated molecular diagnostic techniques. Second, laboratories need to be able to implement the most commonly utilized molecular methods, such as immunohistochemistry and techniques such as fluorescent in situ hybridization and quantitative polymerase chain reaction. Third, laboratories need to be able to analyze and display complex data sets and to provide an interpretation that complements other diagnostic techniques (e.g. clinical and laboratory information, diagnostic imaging and conventional histopathology). Large referral centers will have the resources to use sophisticated analytical technologies, but genomic and proteomic second or third generation tests derived from comprehensive data sets will not be available at primary care institutions. It is likely that large reference laboratories will do much of the analytical work on cells and tissues and will return results to a clinic where multidisciplinary teams will administer the optimal therapy. The treating team will ensure optimal follow-up, using a panel of predictive and personalized tests based on the genetic and epigenetic characteristics of host and tumor cells. Tests detecting the earliest emergence of resistant cells will enable therapists to adapt the therapy to target incipient clonal expansion of newly evolved tumor cells. It is hoped that by hitting the right spot and hitting it hard we can vastly improve survival even if we are a step behind the tumor cells! 